\title{
Modulation of FoxO1 Expression by miR- 21 to Promote Growth of Pancreatic Ductal Adenocarcinoma
}

\author{
Weifeng Song ${ }^{a, b}$ Qi Li ${ }^{a, b}$ Lei Wang ${ }^{a}$ Liwei Wang ${ }^{a, b}$ \\ aDepartment of Medical Oncology, Shanghai First People's Hospital, Shanghai Jiao Tong University, \\ bShanghai Key Laboratory of Pancreatic Diseases Research, Shanghai, China
}

\section{Key Words}

Pancreatic ductal adenocarcinoma $•$ FoxO1 $\bullet$ miR-21

\begin{abstract}
Background: Pancreatic ductal adenocarcinoma (PDAC) is one of the most lethal primary tumors in humans, with undetermined tumorigenesis. Although previous work by us, and by others, has clearly demonstrated an involvement of miR-21 in the growth of PDAC, the underlying mechanism has not been clarified. Methods: Here we analyzed the regulation of FoxO1 by miR-21 in vitro and in vivo, using luciferase-reporter assay and pancreatic intraductal infusion of antisense of miR-21, respectively. Results: We found that overexpression of miR-21 in PDAC cells decreased FoxO1 protein levels, whereas inhibition of miR-21 increased FoxO1 levels. Further, miR-21 bound to FoxO1 mRNA to prevent its translation through its $3^{\prime} U T R$. Moreover, administration of antisense of miR-21 through an intraductal infusion system significantly decreased miR-21 levels and increased FoxO1 levels in implanted PDAC, resulting in a significant decrease in PDAC growth. Conclusion: Taken together, our data highlight miR21/FoxO1 axis as a novel therapeutic target for inhibiting the growth of PDAC.
\end{abstract}

Copyright $(2015$ S. Karger AG, Basel

\section{Introduction}

Pancreatic ductal adenocarcinoma (PDAC) is extremely lethal and the overall median survival of PDAC is less than half a year [1]. In addition, unlike other cancers in the digestive system, molecular-targeted therapies have so far failed to improve patient survival in PDAC, largely resulting from our poor understanding of the tumorigenesis of PDAC [1].

The expression of microRNAs (miRNAs) has been detected in various tumors. Despite of consisting about 22 nucleotides, miRNAs play a substantial role in the regulation of protein-

Liwei Wang

KARGER 125
Department of Medical Oncology, Shanghai First People's Hospital, Shanghai Jiao Tong University, 100 Haining Road, Shanghai 200080 (China)

Tel. +862163240090-3945, Fax +862163069779, E-Mail qi_li14@163.com 
Song et al.: miR-21 Promotes PDAC Growth Through FoxO1 Inhibition

coding gene expression, as well as various cellular events including proliferation, apoptosis, and differentiation [2-4]. Previous work has shown that aberrant miRNA expression could impact normal biological processes in pancreatic cells, resulting in tumor initiation and progression. Specifically, microRNA-21 (miR-21) has been highlighted as a major aberrantly expressed miRNAs in early pancreatic cancer lesions and pancreatic tumors [24]. Nevertheless, the mechanism by which miR-21 regulates the growth of PDAC is still not completely understood.

Forkhead box protein (Fox) proteins are a subgroup of the Forkhead family of transcription factors, characterized by a conserved DNA-binding domain. Fox proteins comprise more than 100 members in humans, classified from FoxA to FoxR on the basis of sequence similarity [5]. These proteins participate in very diverse functions. Fox01 transcription factors orchestrate various cellular events like apoptosis and cell-cycle control [5]. However, whether FoxO1 may be involved in the miR-21-mediated increases in PDAC growth is unknown.

Here we report that miR-21 bound Fox01 mRNA to prevent its translation through its 3'UTR. Moreover, administration of antisense of miR-21 through a pancreatic intraductal infusion system significantly decreased miR-21 levels and increased FoxO1 levels in implanted PDAC, resulting in a significant decrease in cancer growth.

\section{Materials and Methods}

Culture of human PDAC cell line

PANC-1 has been generated from a human carcinoma of the exocrine pancreas in 1975 [6], and was purchased from ATCC. PANC-1 was cultured in Dulbecco's modified Eagle's medium (DMEM) supplemented with $20 \%$ fetal bovine serum (Invitrogen, Carlsbad, CA, USA).

\section{Transfection of PANC-1 cells}

PANC-1 cells were transfected with a miR-21 construct, or an antisense of miR-21, or control scrambled plasmid, and selected for transduced cells based on green fluorescence protein (GFP) to generate PANC-1-miR-21, or PANC-1-ctl, or PANC-1-as-miR-21 cells respectively. miR-21 sequence: 5'-UAGCUUAUCAGACUGAUGUUGA-3', miR-21 antisense sequence: 5'-UCAACAUCAGUCUGAUAAGCUA-3', control sequence: 5'-UUGUACUACACAAAAGUAAUG-3'. PANC-1 cells were also transfected with a plasmid with luciferase and GFP construct, to allow in vivo tracing and isolation from the receipt pancreas by flow cytometry, respectively. Transfection was performed with Lipofectamine 2000 reagent (Invitrogen, USA), according to the manufacturer's instructions.

\section{Luciferase-reporter activity assay}

Luciferase-reporters were successfully constructed using molecular cloning technology. Target sequence was inserted into pGL3-Basic vector (Promega, USA) to obtain pGL3-Fox01-3'UTR, which contains the miR-21 binding sequence (Fox01-3'UTR sequence) as follows: AAGCCAGCUCUAUUGUAAGCUU. PANC1-miR-21, or PANC-1-ctl, or PANC-1-antisense (as)-miR-21 cells were seeded in 24-well plates for 24 hours, after which they were transfected with $1 \mu \mathrm{g}$ of Luciferase-reporter plasmids per well using PEI Transfection Reagent. Then luciferase activities were measured using the dual-luciferase reporter gene assay kit (Promega, USA), according to the manufacturer's instructions.

\section{Intraductal infusion}

Intraductal infusion was performed as has been previously described [7-12]. Briefly, the duodenum was isolated to expose the common bile duct, after which a microclamp was placed on the common bile duct above the branching of the pancreatic duct. A 31-gauge blunt-ended catheter was then put into the common bile duct through the sphincter of Oddi in the duodenum, which was then clamped with another microclamp to prevent backflow. The other end of the catheter is connected to a micro-infusion apparatus, which delivers $100 \mu \mathrm{l}$ of $1 \mathrm{mg}$ antisense of miR-21, or control saline, via the catheter at a rate of $5 \mu \mathrm{l} / \mathrm{min}$. After infusion, the hole created by the catheter in the duodenum was closed with 6-0 gauge suture. 
In vivo tumor model and imaging of the implanted tumor by bioluminescence

Luciferase-carrying PANC-1 cells (PANC-1-LUC-GFP) of $10^{6}$ were directly injected into the parenchyma of the pancreas in NOD/SCID mice, as has been described before [13, 14]. The tumor growth in the living animals was monitored and quantified by luminescence levels. Bioluminescence was measured with the IVIS imaging system (Xenogen Corp., Alameda, CA). All of the images were taken 10 minutes after intraperitoneal injection of luciferin (Sigma) of $150 \mathrm{mg} / \mathrm{kg}$ body weight, as a 60-second acquisition and 10 of binning. During image acquisition, mice were sedated continuously via inhalation of $3 \%$ isoflurane. Image analysis and bioluminescent quantification was performed using Living Image software (Xenogen Corp).

\section{Pancreas digestion and isolation of implanted PANC-1 cell by flow cytometry}

The mouse pancreas was first perfused with $35 \mathrm{mg} / \mathrm{dl}$ collagenase (Sigma) from the common bile duct, then was either completely digested with $35 \mathrm{mg} / \mathrm{dl}$ collagenase for 45 minutes to isolate implanted PANC-1 cells by flow cytometry, based on their expression of GFP.

\section{Quantitative PCR (RT-qPCR)}

MiRNA and total RNA were extracted from cultured cells with miRNeasy mini kit or RNeasy kit (Qiagen, Hilden, Germany), respectively, and used for cDNA synthesis. Quantitative PCR was performed in duplicates with QuantiTect SYBR Green PCR Kit (Qiagen). All primers were purchased from Qiagen. Values of genes were normalized against $\alpha$-tubulin, and then compared to controls.

\section{Statistical analysis}

All statistical analyses were carried out using the SPSS 17.0 statistical software package. All values are depicted as mean \pm standard deviation and are considered significant if $\mathrm{p}<0.05$. All data were statistically analyzed using one-way ANOVA with a Bonferoni correction.

\section{Results}

MiR-21 decreased FoxO1 transcripts in vitro

We have recently reported significantly higher levels of miR-21 in PDAC. Here we aimed to figure out the mechanism underlying the regulation of PDAC growth by miR-21. We used a human PDAC cell line, PANC-1, and overexpressed or inhibited miR-21 expression in these cells. First, modulation of miR-21 levels in PANC-1 cells was confirmed by RT-qPCR (Fig. 1A). Since FoxO1 has been shown to play a critical role in cell-cycle control, and specifically express in pancreatic cells, we were thus prompted to evaluate whether it may be involved

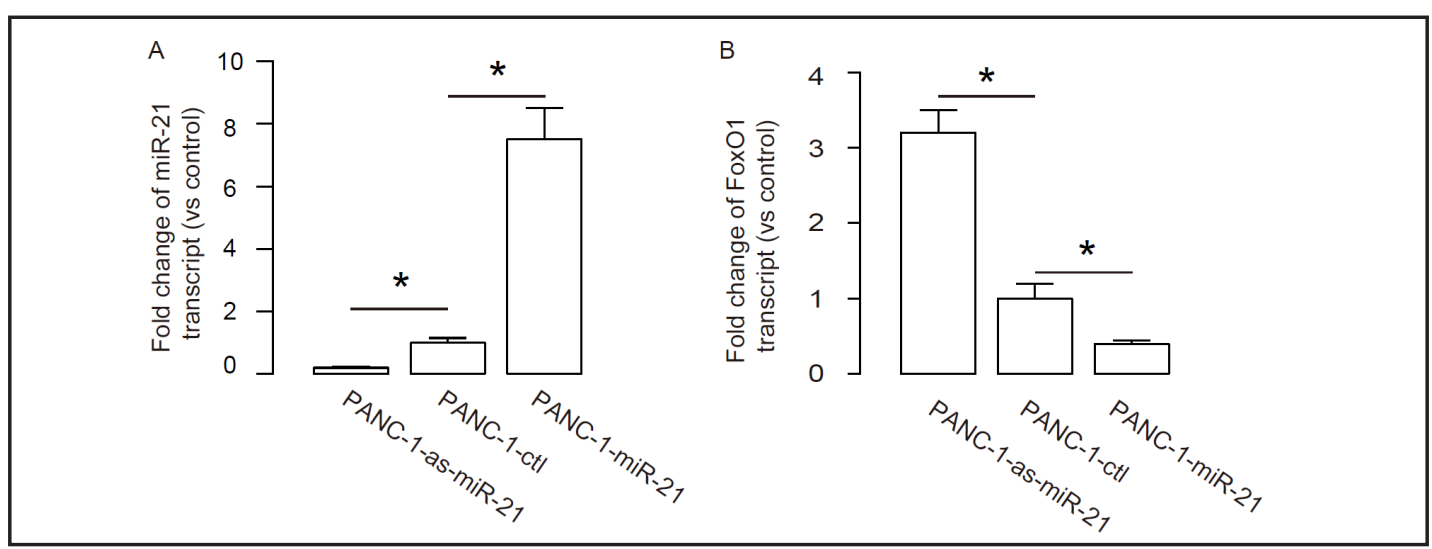

Fig. 1. MiR-21 inhibited expression of Fox01 in PDAC cells. We overexpressed or inhibited miR-21 expression in a human PDAC cell line, PANC-1, to get PANC-1-miR-21 and PANC-1-as-miR-21 cells, respectively. The cells transduced with control plasmids were used as controls (PANC-1-ctl). (A-B) RT-qPCR on miR-21 (A) and Fox01 (B). *: p<0.05. 
Fig. 2. MiR-21 targeted 3'UTR of Fox01 to inhibit its expression. (A) Bioinformatics analysis of Fox01 target sequence show that the miR-21 binding sites in the FoxO1 mRNA sequence 3'UTR ranged from 1975th base site to 1996th base site. (B) PANC-1-miR-21, PANC-1-ctl and PANC-1as-miR-21 cells were then seeded in 24-well plates for 24 hours, after which they were transfected with $1 \mu \mathrm{g}$ of Fox01-3'UTR luciferase-reporter plasmid. The luciferase activities in these cells were then evaluated. *: $\mathrm{p}<0.05$.

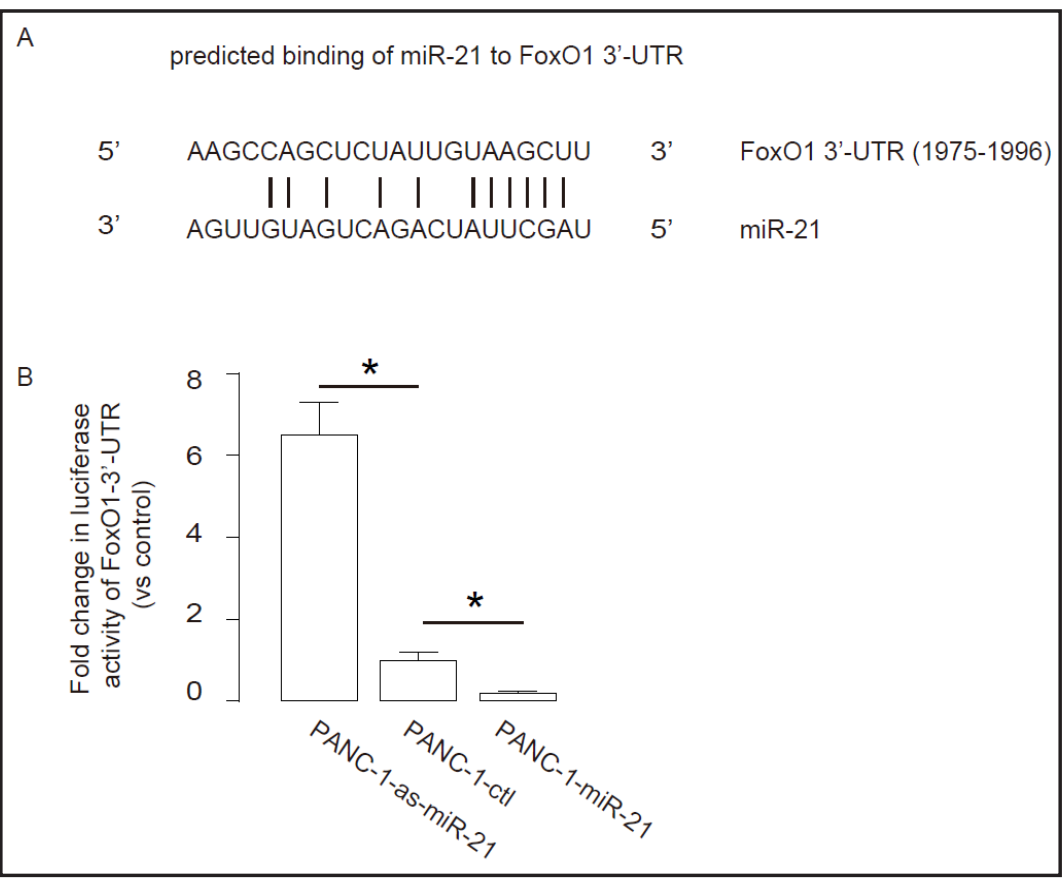

in the miR-21-regulated PDAC cell growth. We found that the expression of FoxO1 in miR21-overexpressing PANC-1 cells was significantly decreased, while the expression of FoxO1 in miR-21-depleted PANC-1 cells was significantly increased (Fig. 1B). These data suggest a possibility that Fox01 expression in PDAC cells may be regulated by miR-21.

MiR-21 targets 3'UTR of FoxO1 to inhibit its expression

Since our data suggest that miR-21 may inhibit FOXO1 expression, we performed bioinformatics analysis of FoxO1 target sequence, which showed that the miR-21 binding sites in the FoxO1 mRNA sequence 3'UTR ranged from 1975th base site to 1996th base site (Fig. 2A). PANC-1-miR-21, PANC-1-ctl and PANC-1-as-miR-21 cells were then seeded in 24-well plates for 24 hours, after which they were transfected with $1 \mu \mathrm{g}$ of Fox01-3'UTR Luciferase-reporter plasmid. We found that the luciferase activities in PANC-1-as-miR-21 cells were significantly higher than the control, while the luciferase activities in PANC-1miR-21 cells were significantly lower than the control (Fig. 2B). These data suggest that MiR21 targets 3'UTR of Fox01 to inhibit its expression.

Intraductal infusion of antisense of miR-21 inhibited PDAC growth in vivo

PANC-1 cells were transfected with a plasmid with luciferase and GFP construct (PANC-1-LUC-GFP), to allow in vivo tracing and isolation from the receipt pancreas by flow cytometry, respectively. We used 20 male NOD/SCID mice that were randomly separated into 2 groups of 10 each. All the mice received intrapancreatic injection of $10^{6}$ PANC-1-LUCGFP cells to form PDAC. One month later, one group of mice received pancreatic intraductal infusion of saline. Another group received pancreatic intraductal infusion of miR-21 antisense for inhibiting miR-21 expression. After 4 weeks, the tumor growth was monitored and quantified by luminescence levels. We found significant decreases in tumor growth in miR-21-antisense-infused mice (Fig. 3A-B), suggesting that pancreatic intraductal infusion of antisense of miR-21 inhibits PDAC growth in vivo.

We then isolated the implanted PDAC cells from the pancreas digests by flow cytometry, based on their expression of GFP (Fig. 4A). We found that the implanted PDAC cells significantly decreased their expression of miR-21, and significantly increased their expression of FoxO1 (Fig. 4B), which confirmed the effect of intraductal infusion of antisense 
A

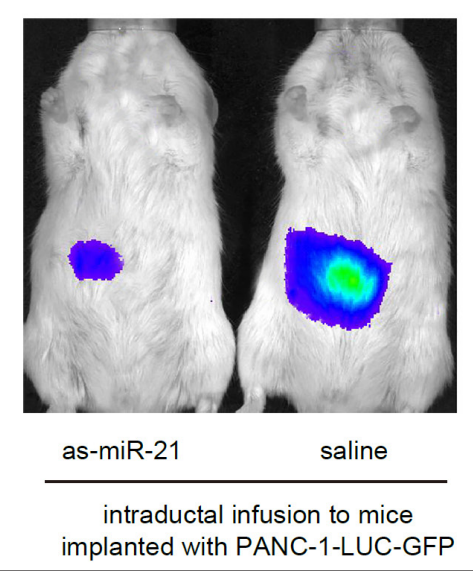

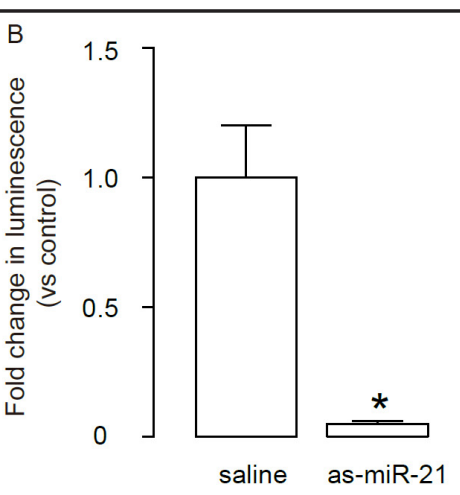

intraductal infusion to mice implanted with PANC-1-LUC-GFP

Fig. 3. Pancreatic intraductal infusion of antisense of miR-21 inhibited PDAC growth in vivo. PANC-1 cells were transfected with a luciferase and GFP -containing plasmid (PANC-1-LUC-GFP), to allow in vivo tracing and isolation from the receipt pancreas by flow cytometry, respectively. NOD/SCID mice received pancreatic intraductal infusion of $10^{6}$ PANC-1-LUC-GFP cells and developed PDAC. One month later, the mice received pancreatic intraductal infusion of either saline (as control), or antisense of miR-21, to inhibit miR-21 expression. After 4 weeks, the tumor growth was monitored and quantified by luminescence levels, shown by representative images (A), and by quantification (B). *: $\mathrm{p}<0.05$.

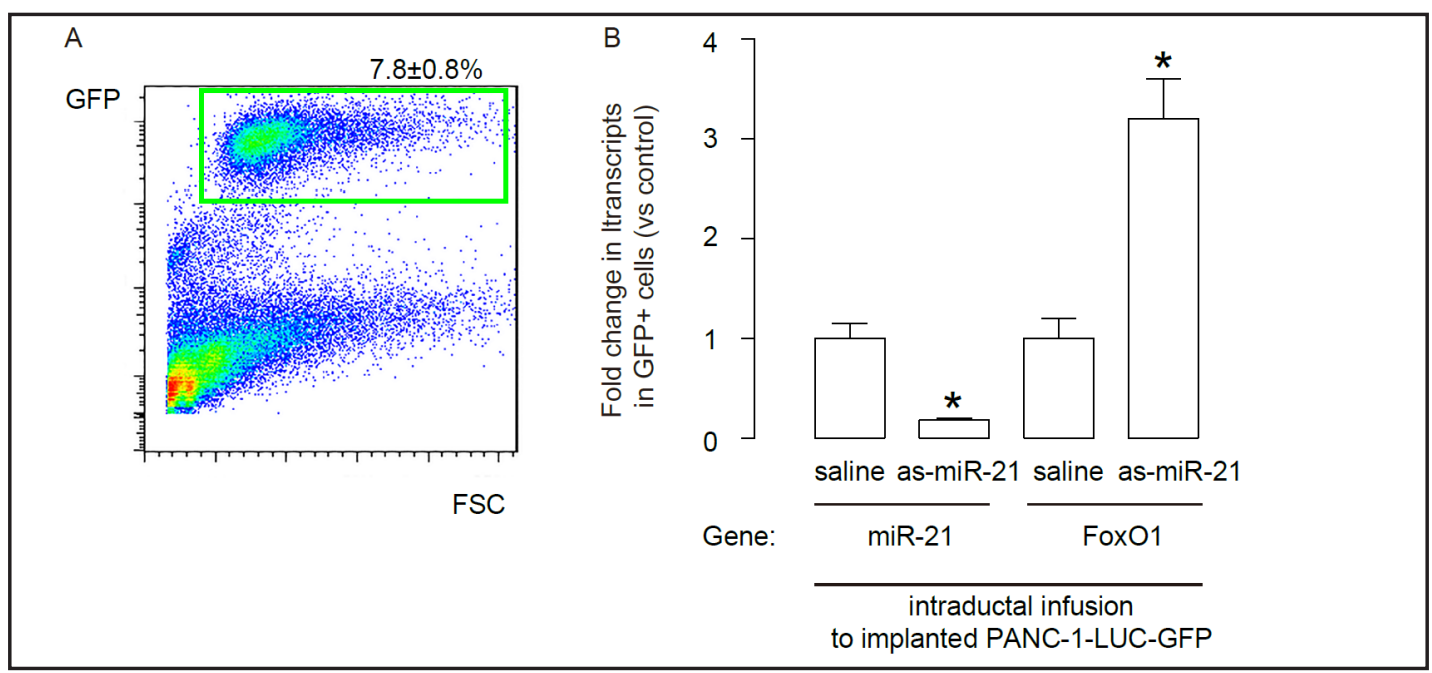

Fig. 4. Pancreatic intraductal infusion of antisense of miR-21 inhibited miR-21 and increased FoxO1 in implanted PDAC cells. (A) The implanted PDAC cells were isolated from the pancreas by flow cytometry, based on their expression of GFP. (B) RT-qPCR on isolated PDAC cells for miR-21 and FoxO1. *: p<0.05.

Fig. 5. Schematic of the model. MiR-21 promotes growth of PDAC through Fox01 inhibition.

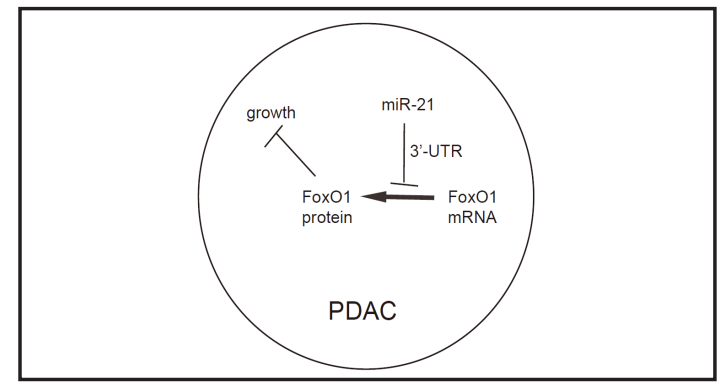

of miR-21. Our study thus demonstrate that miR-21 promotes the growth of PDAC through Fox01 inhibition (Fig. 5). 
Song et al.: miR-21 Promotes PDAC Growth Through FoxO1 Inhibition

\section{Discussion}

Previous reports have highlighted an essential role of miR-21 in the pathogenesis of PDAC. In this study, we aimed to understand the molecular mechanisms that underlie the regulation of the tumorigenesis of PDAC by miR-21. Then we used human PDAC cell lines to study the mechanism. We examined several lines and essentially obtained similar results. Here we only showed our data using PANC-1 cells.

Although miR-21 has been shown to promote angiogenesis [15-18], and angiogenic factors have been shown to be produced by both normal duct cells and PDAC [7, 19-21], here we just focused on the direct targets downstream of miR-21 in controlling cell growth. Since Fox01 plays a critical role in cell-cycle control and has been shown to express in pancreatic cells, we thus examined whether miR-21 may inhibit the expression of Fox01. We either overexpressed or inhibited miR-21 expression in PDAC cells, which decreased or increased the expression of Fox01, respectively. These data suggest that Fox01 expression in PDAC cells is regulated by miR-21. Moreover, using luciferase reporter assay, we figured out the direct binding site of miR-21 at 3'UTR on Fox01 mRNA. Taken together, these findings strongly demonstrate a critical role of miR-21 in regulating FoxO1 transcription, rather than regulating the degradation of mRNA. FoxO1 is a protein that is modified by phosphorylation, acetylation and ubiquitination and binding protein partners, which potentially affect its function. Here we did not study these protein modification by miR-21. Future attempts should be taken to address these questions to completely understand the relationship between Fox01 and miR-21.

In an in vivo loss-of-function experiment, we treated implanted PDAC cells with antisense of miR-21. The alterations in miR-21 and Fox01 levels confirmed our findings in vitro. Of note, miR-21 inhibition in PDAC cells significantly inhibited their growth in vivo.

Our findings highlight miR-21/Fox01 axis as a novel therapeutic target for inhibiting the growth of PDAC. In future, experiments should be designed to examine whether miR-21 may affect PDAC cell invasion through activation of angiogenic factors, e.g. vascular endothelial cell growth factor, MMPs. These approaches may help to fully understand the role of miR-21 in the tumorigenesis of PDAC.

\section{Disclosure Statement}

The authors have declared that no competing interests exist.

\section{Reference}

1 Han H, Von Hoff DD: Snapshot: Pancreatic cancer. Cancer Cell 2013;23:424-424, e421.

2 Sicard F, Gayral M, Lulka H, Buscail L, Cordelier P: Targeting mir-21 for the therapy of pancreatic cancer. Mol Ther 2013;21:986-994.

-3 Tavano F, di Mola FF, Piepoli A, Panza A, Copetti M, Burbaci FP, Latiano T, Pellegrini F, Maiello E, Andriulli A, di Sebastiano P: Changes in mir-143 and mir-21 expression and clinicopathological correlations in pancreatic cancers. Pancreas 2012;41:1280-1284.

-4 Ali S, Ahmad A, Banerjee S, Padhye S, Dominiak K, Schaffert JM, Wang Z, Philip PA, Sarkar FH: Gemcitabine sensitivity can be induced in pancreatic cancer cells through modulation of mir-200 and mir-21 expression by curcumin or its analogue cdf. Cancer Res 2010;70:3606-3617.

5 Gross DN, van den Heuvel AP, Birnbaum MJ: The role of foxo in the regulation of metabolism. Oncogene 2008;27:2320-2336.

6 Lieber M, Mazzetta J, Nelson-Rees W, Kaplan M, Todaro G: Establishment of a continuous tumor-cell line (panc-1) from a human carcinoma of the exocrine pancreas. Int J Cancer 1975;15:741-747. 
Song et al.: miR-21 Promotes PDAC Growth Through FoxO1 Inhibition

7 Xiao X, Prasadan K, Guo P, El-Gohary Y, Fischbach S, Wiersch J, Gaffar I, Shiota C, Gittes GK: Pancreatic duct cells as a source of vegf in mice. Diabetologia 2014;57:991-1000.

8 Xiao X, Guo P, Shiota C, Prasadan K, El-Gohary Y, Wiersch J, Gaffar I, Gittes GK: Neurogenin3 activation is not sufficient to direct duct-to-beta cell transdifferentiation in the adult pancreas. J Biol Chem 2013;288:25297-25308.

-9 Xiao X, Gaffar I, Guo P, Wiersch J, Fischbach S, Peirish L, Song Z, El-Gohary Y, Prasadan K, Shiota C, Gittes GK: M2 macrophages promote beta-cell proliferation by up-regulation of smad7. Proc Natl Acad Sci U S A 2014;111:E1211-1220.

$\longrightarrow 10$ Jimenez V, Ayuso E, Mallol C, Agudo J, Casellas A, Obach M, Munoz S, Salavert A, Bosch F: In vivo genetic engineering of murine pancreatic beta cells mediated by single-stranded adeno-associated viral vectors of serotypes 6, 8 and 9. Diabetologia 2011;54:1075-1086.

-11 Wang Z, Zhu T, Rehman KK, Bertera S, Zhang J, Chen C, Papworth G, Watkins S, Trucco M, Robbins PD, Li J, Xiao X: Widespread and stable pancreatic gene transfer by adeno-associated virus vectors via different routes. Diabetes 2006;55:875-884.

12 Xiao X, Guo P, Prasadan K, Shiota C, Peirish L, Fischbach S, Song Z, Gaffar I, Wiersch J, El-Gohary Y, Husain SZ, Gittes GK: Pancreatic cell tracing, lineage tagging and targeted genetic manipulations in multiple cell types using pancreatic ductal infusion of adeno-associated viral vectors and/or cell-tagging dyes. Nat Protoc 2014;9:2719-2724.

13 Cao X, Han ZB, Zhao H, Liu Q: Transplantation of mesenchymal stem cells recruits trophic macrophages to induce pancreatic beta cell regeneration in diabetic mice. Int J Biochem Cell Biol 2014;53:372-379.

14 Zhou Q Brown J, Kanarek A, Rajagopal J, Melton DA: In vivo reprogramming of adult pancreatic exocrine cells to beta-cells. Nature 2008;455:627-632.

15 Zhao Y, Xu Y, Luo F, Xu W, Wang B, Pang Y, Zhou J, Wang X, Liu Q: Angiogenesis, mediated by mir-21, is involved arsenite-induced carcinogenesis. Toxicol Lett 2013;223:35-41.

16 Zhao D, Tu Y, Wan L, Bu L, Huang T, Sun X, Wang K, Shen B: In vivo monitoring of angiogenesis inhibition via down-regulation of mir-21 in a vegfr2-luc murine breast cancer model using bioluminescent imaging. PLoS One 2013;8:e71472.

-17 Zaravinos A, Radojicic J, Lambrou GI, Volanis D, Delakas D, Stathopoulos EN, Spandidos DA: Expression of mirnas involved in angiogenesis, tumor cell proliferation, tumor suppressor inhibition, epithelialmesenchymal transition and activation of metastasis in bladder cancer. J Urol 2012;188:615-623.

18 Liu LZ, Li C, Chen Q, Jing Y, Carpenter R, Jiang Y, Kung HF, Lai L, Jiang BH: Mir-21 induced angiogenesis through akt and erk activation and hif-1alpha expression. PLoS One 2011;6:e19139.

19 Movahedi B, Gysemans C, Jacobs-Tulleneers-Thevissen D, Mathieu C, Pipeleers D: Pancreatic duct cells in human islet cell preparations are a source of angiogenic cytokines interleukin-8 and vascular endothelial growth factor. Diabetes 2008;57:2128-2136.

20 Schulz P, Fischer C, Detjen KM, Rieke S, Hilfenhaus G, von Marschall Z, Bohmig M, Koch I, Kehrberger J, Hauff P, Thierauch KH, Alves F, Wiedenmann B, Scholz A: Angiopoietin-2 drives lymphatic metastasis of pancreatic cancer. FASEB J 2011;25:3325-3335.

-21 Bausch D, Pausch T, Krauss T, Hopt UT, Fernandez-del-Castillo C, Warshaw AL, Thayer SP, Keck T: Neutrophil granulocyte derived mmp-9 is a vegf independent functional component of the angiogenic switch in pancreatic ductal adenocarcinoma. Angiogenesis 2011;14:235-243. 